\title{
Corela
}

Cognition, représentation, langage

HS-19 | 2016

Le point de vue pris au mot

\section{Les points de vue comme strate interprétative}

\section{François Nemo}

\section{(2) OpenEdition}

\section{Journals}

Édition électronique

URL : http://journals.openedition.org/corela/4301

DOI : 10.4000/corela.4301

ISSN : $1638-573 X$

Éditeur

Cercle linguistique du Centre et de l'Ouest - CerLICO

\section{Référence électronique}

François Nemo, "Les points de vue comme strate interprétative », Corela [En ligne], HS-19 | 2016, mis en ligne le 08 juin 2016, consulté le 21 avril 2019. URL : http://journals.openedition.org/corela/4301 ; DOI : 10.4000/corela.4301

Ce document a été généré automatiquement le 21 avril 2019.

\section{(c) (i) (9)(2)}

Corela - cognition, représentation, langage est mis à disposition selon les termes de la licence Creative Commons Attribution - Pas d'Utilisation Commerciale - Partage dans les Mêmes Conditions 4.0 International. 


\title{
Les points de vue comme strate interprétative
}

\author{
François Nemo
}

\section{Introduction}

1 Le travail scientifique de Pierre-Yves Raccah est de part en part attaché aux notions d'argumentation, de points de vue et d'idéologie, à condition de comprendre que ces notions sont définies en tant que concepts linguistiques et surtout en tant qu'outils, posés comme indispensables, de toute description sémantique de la langue et des langues (et en premier lieu du lexique et des mots ${ }^{1}$ ).

2 Ce faisant, comme tous ceux qui, dans le cadre des linguistiques énonciatives, ont à la suite de Benveniste accepté l'idée d'une cristallisation du discours en langue, puis à la suite de Ducrot celle d'une sémantique intégrant la pragmatique (en tant que signes et phrases contribuent en la contraignant à la détermination de la valeur pragmatique des énoncés) et devant donc intégrer pleinement la dimension argumentative des énoncés, il s'est retrouvé avec d'autres dont je suis à être confronté à un double défi :

- convaincre un champ divisé de ce point de vue de l'utilité et de l'importance

d'une approche argumentative en sémantique linguistique ;

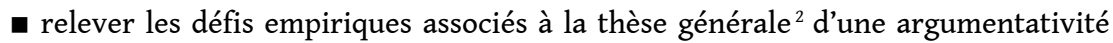
de la langue, notamment celui de situer en langue la forme et le niveau d'inscription de celle-ci.

3 Si en effet la liste est devenue très longue des phénomènes sémantiques et linguistiques que les approches non-argumentatives ne sont pas à même d'expliquer, le fonctionnement collectif de la sémantique comme discipline fait qu'elle s'est structurée assez largement depuis le début des années 70 autour de théories ayant en commun de mettre en avant des dimensions différentes de la réalité sémantique ainsi que des observables différents. Avec comme résultat, comme je le signalais déjà en 1992, de jouer souvent les données, en tant qu'elles donnent accès à des mécanismes différents, les unes contre les autres : 
À l'heure actuelle, il existe en fait deux types de sémantiques: celles qui ne prennent pas en compte l'existence d'une orientation argumentative des énoncés et celles qui, sous une forme plus ou moins radicale (i.e. selon le degré de reconnaissance accordé à l'aspect informatif du langage), considèrent celles-ci comme devant constituer le point de départ obligé de toute théorie sémantique. Il y a donc des sémantiques du « rien argumentatif » comme il y a des sémantiques du

« tout argumentatif ». (Nemo $1992: 122)$

La situation n'a de fait que partiellement changé depuis, même si l'histoire de la notion de point de vue depuis trente ans a montré la capacité de certains outils théoriques techniquement nécessaires et heuristiquement neutres, à s'imposer dans de nombreux domaines de la sémantique, comme par exemple la sémantique des temps et des modes (Gosselin 1996 ; Rabatel 1998). Et ce y compris il faut le souligner, dans la façon dont le développement parallèle des théories de l'argumentation dans la langue et des théories de la polyphonie a pu conduire à un recours progressif à la notion de point de vue dans les deux cas.

5 Comme mon titre l'indique, mon objectif ultime va être de montrer ici qu'au-delà des fonctionnements collectifs de la période concernée, ce que ces quarante d'ans d'histoire de la sémantique et en particulier le travail de Pierre-Yves Raccah ont permis d'établir, c'est l'existence d'une strate interprétative spécifique, linguistiquement construite pour une large part, et qui concerne le marquage et la lexicalisation des points de vue et leurs rapports au monde ${ }^{3}$.

6 Ce qui revient en quelque sorte à poser que si le sens n'est en réalité ni conceptuel ni procédural, c'est avant tout ${ }^{4}$ parce que l'interprétation ne manipule pas des concepts mais des conceptions du monde et que même si les conceptions du monde portent par définition sur le monde, elles imposent pour employer une formulation chère à PierreYves Raccah d'admettre certaines choses pour être comprises et sont donc de ce fait porteuses de ce qu'il nomme points de vue et idéologie.

7 Si en effet une partie des conceptions du monde en question s'inscrivent dans un rapport aux choses que Pierre Cadiot et moi-même avions théorisé sous le nom de propriétés extrinsèques (Cadiot et Nemo 1997) en notant que le monde auquel les langues humaines renvoient n'est pas un monde sans l'homme mais un monde avec l'homme, il s'ensuit inévitablement que les descripteurs ${ }^{5}$ linguistiques ne peuvent pas ne pas parler autant de la relation aux choses que des choses qu'ils décrivent dans le cadre de cette relation ${ }^{6}$. On aura ainsi : ce que je résumais il y a longtemps déjà (Nemo 1992 : 286) par la formule :

- intenté ${ }^{7} \mathrm{x}$ (rapport à l'intenté) = descripteur

10 Et ceci alors beaucoup de sémantiques et même de définitions de la sémantique supposent que celle-ci peut être neg-anthropique et postulent donc une relation directe entre mots (ou phrases) et monde censé ne pas être polluée $e^{8}$ par le fait que les objets et le monde existent aussi dans la relation qu'ils ont avec les hommes.

11 Mais avant de revenir longuement sur la façon dont l'interprétation des descripteurs varie en fonction des points de vue qui leur sont associés, ou encore sur l'existence de descripteurs prosodiques qui rendent à chaque instant explicite le rapport du locuteur (et parfois des interlocuteurs) à ce dont il est question, il va m'être indispensable d'expliquer ce que j'entends par strate interprétative, et donc d'introduire la notion de plurisémie et expliquer ce qui fait que quelque chose comme l'association aux énoncés de points de vue 
(et autres biais attentionnels) peut concerner tous les énoncés et constituer de ce fait une strate interprétative à part entière (Nemo 2014).

\section{Plurisémie et sémantique}

12 Le terme de plurisémie (Nemo, Petit et Portuguès 2012) ne recouvre pas la notion de polysémie, puisqu'il ne concerne pas la diversité des sens des signes linguistiques mais la diversité des strates interprétatives associées (y compris lexicalement) à chaque emploi d'un signe'.

13 Localement, elle vise à décrire par exemple le fait que si un mot comme " rouge-gorge »accidentellement monosémique en français - a bien la valeur dénominative que lui prêtent les dictionnaires, à savoir d'être le nom d'un certain type d'oiseau, ce n'est analytiquement que le résultat du fait que l'expression a été employée à propos d'un oiseau. Ce qui fait de cette valeur à la fois indexicale et lexicalisée non pas le sens du mot seulement une des strates interprétatives associées à celui-ci, qui en contient au moins quatre :

- le fait que «rouge-gorge » veuille d'abord dire "gorge-rouge » par héritage lexical ;

- le fait que la construction soit interprétée comme exocentrique ( $\mathrm{X}$ à gorge rouge) par héritage constructionnel;

- le fait que X s'avère indexicalement (et accidentellement) être un oiseau, par héritage indexical ;

- le fait que dire «rouge-gorge» impose obligatoirement une contrainte de type «non-gorge non-rouge », autrement dit le fait qu'un oiseau tout rouge ne pourra pas être appelé «rouge-gorge " même s'il vérifie parfaitement la condition de vérité « $\mathrm{X}$ a la gorge rouge». Le tout par héritage d'une contrainte contributionnelle $^{10}$ (Nemo, Petit et Portuguès 2012).

14 La notion de plurisémie vise aussi théoriquement à sortir de la façon dont les définitions formelles de la grammaire quand elles définissent le lexique comme une liste d'items $a, b$, $c$, $d$, etc. supposent que ceux-ci seront monosémiques et unisémiques ${ }^{11}$, avec un sens rêvé comme réductible à sa seul valeur référentielle, et avec en conséquence une forte propension à prôner systématiquement le dégroupement homonymique (Fodor et Lepore 1998).

Plus globalement, elle recouvre des distinctions aussi classiques que les distinctions entre :

- sinn et bedeutung, sens et référence ;

- signification et sens, signification et désignation (Benveniste) ;

- valeur dénotative et valeur connotative ;

- valeurs locutoire, illocutoire, perlocutoire;

- valeur argumentative et valeur informative ;

sans pour autant être réductible à ces notions très générales, ni en particulier aux conceptions presque toujours bistratiques de la plurisémie qui comme nous le verrons en limitent la portée et peinent à rendre compte de la complexité des faits sémantiques.

17 Le plus souvent en effet, parce que la plurisémie intervient avant tout en tant qu'hypothèse interne des théories sémantiques concernées, autrement dit comme explication, elle n'est pas conçue comme une hypothèse externe, autrement dit comme un observable dont l'analyse devrait par définition être ouverte. 
en tant que phénomène empirique, la plurisémie s'avère omniprésente, incontournable et nettement plus pluristratique que l'on pourrait le penser (Nemo, Petit et Portuguès 2012; Nemo 2014; Petit et Nemo 2015). Ainsi les travaux sur enfin (Petit 2009; Nemo et Petit 2009; Nemo et Petit 2012) ont montré en français, la nécessité de distinguer au moins trois strates sémantiques, à savoir :

- la strate morphémique, qui est celle de l'indication codée par le morphème enfin ;

- la strate de profilage sémantique, dans laquelle sont instanciées des variables non déterminées par la strate morphémique ;

- la strate de profilage prosodique, qui marque le rapport à la situation.

Chacune de ces strates étant obligatoire pour rendre compte de la polysémie de enfin. Plus généralement, ce type d'observation comme toutes celles que je serai amené à présenter, implique minimalement :

- de décrire et théoriser la plurisémie comme un véritable objet empirique ;

- de demander à toutes les théories sémantiques de rendre explicite leur conception de la plurisémie, et en particulier (et de façon argumentée) leur position par rapport à l'existence de telle ou telle strate ;

- de ne pas chercher à hiérarchiser les strates entre elles, ni a fortiori à en ignorer, une science « exacte » n'ayant pas à trier les dimensions du réel dont elle a à rendre compte.

Le point le plus important car le plus grave en ce qui concerne la plurisémie est en effet que si comme c'est le cas il y a plurisémie et si les théories sémantiques ne sont pas plurisémistes, alors on peut prévoir qu'il y aura pendant longtemps autant de théories apparemment concurrentes « $d u$ » sens linguistique qu'il y a de strates sémantiques. Mais aussi qu'il y aura entre ces théories ${ }^{12}$, autant de revendications pour cette strate d'un statut central ou fondamental en sémantique. Nous retombons là sur le problème signalé dans l'introduction et qui conduit en pratique les sémanticiens à jouer les strates les unes contre les autres et donc à ignorer délibérément des strates dont l'existence ne fait pourtant aucun doute. Ce qui fait d'une certaine façon de l'étude de la plurisémie le chantier le plus important et le plus central de toute sémantique et que ce qui rend donc nécessaire de faire de l'explicitation de sa conception de la plurisémie une composante obligatoire du cahier des charges de toute théorie sémantique ${ }^{13}$.

\subsection{Plurisémie et linguistiques de l'énonciation}

21 Sans pouvoir en développer tous les aspects, il va être souhaitable de rendre explicite ici la conception en quelque sorte cyclique de la plurisémie qui est celle des linguistiques de l'énonciation, sachant que certains aspects de cette conception ont constitué la toile de fond du travail théorique de Pierre-Yves Raccah, notamment quand il s'agit de comprendre dans celui-ci de quoi « langue » est le nom.

La célèbre formule de Benveniste selon laquelle "nihil est in lingua quod non prius fuerit in oratione» (Rien n'est dans la langue qui n'ait d'abord été dans l'énonciation), ainsi que la notion de cristallisation du discours en langue, traduisent en effet la façon dont est conçu génétiquement le rapport entre strates interprétatives.

23 Ainsi les interprétations pragmatiques de l'énoncé «j'ai à faire ", à savoir le fait que l'énonciateur ne souhaite pas dire ce qu'il fait (parce que faire des affaires n'est pas honorable dans la cité grecque), vont-elles se cristalliser en langue pour devenir le nom même de ce que l'on ne souhaite pas nommer mais aussi dans les emplois nondénominatifs pour exprimer les points de vue associés à l'emploi initial. Ce qui permettra 
par exemple à un énoncé comme « c'est pas tes affaires » de continuer à être porteur du " cela ne te regarde pas » associé à celui-ci ${ }^{14}$.

La langue devient ainsi le réceptacle de l'interprétation plurisémique d'une énonciation mais impose aussi celle-ci dans les énonciations à venir, ce qui rend alors pertinente la définition que Pierre-Yves Raccah donne de la dimension idéologique ce que les mots apportent dans énoncé, à savoir d'obliger l'interprétant à admettre certaines choses pour comprendre ce qui est dit.

$\mathrm{Au}$ total, on a donc chez Benveniste une plurisémie en quelque sorte cyclique, les strates les plus pragmatiques se trouvant génétiquement en amont de la langue, mais la langue (et donc la sémantique) se trouvant elle fonctionnellement première quand elle réactive en emploi les points de vue cristallisés dans les mots.

\subsection{Plurisémie et linguistique des contraintes interprétatives}

La représentation que Benveniste a ainsi donnée des strates interprétatives et de leurs relations en diachronie et en synchronie est une représentation exigeante et qui n'est donc pas toujours opérationnelle dans le travail de description sémantique de signes linguistiques particuliers. Cette représentation n'est d'ailleurs pas sa seule contribution à une théorie de la plurisémie puisqu'il a aussi été à l'origine et de la distinction plurisémiste entre signification et sens et d'une conception très particulière de la relation en diachronie et en synchronie entre ces deux strates.

En démontrant en effet qu'il était possible le cas échéant d'étudier l'ensemble des emplois d'un signe au travers du temps mais aussi dans un ensemble de langues et d'isoler une signification constante (et non intuitive), Benveniste a en effet ouvert la question de savoir comment diable une signification constante pouvait bien traverser le temps et la diversité des langues, et ce sur de très longues durées.

Or s'il a en effet été ainsi à l'origine des sémantiques de la signification puis des sémantiques instructionnelles, indicationnelles ou indexicales, il l'a été aussi et surtout à l'origine de la démonstration progressive du fait que si la signification pouvait servir de déclencheur au processus interprétatif, c'était dans la mesure où elle imposait des contraintes à satisfaire et que la diversité des interprétations et sens lexicaux n'étaient que des formes différentes de satisfaction de ces contraintes. Constat que j'ai souvent résumé en disant que la signification était au sens ce qu'une équation est aux points qui la satisfont, et qui, quand il est associé à un travail sur corpus, conduit à devoir concevoir l'interprétation comme mettant en jeu un ensemble de contraintes à satisfaire, certaines étant des contraintes linguistiques et d'autres non.

Moyennant quoi le problème n'est pas tant de décrire la façon dont dans des mots donnés les interprétations résultant d'une forme particulière d'association entre ces contraintes vont se lexicaliser / cristalliser que d'identifier une à une et séparément les contraintes en question. Un peu comme si dans l'étude de la musique, on passait de la description de l'ensemble des accords de guitare à celle de la guitare elle-même, et notamment de chaque corde et des valeurs qui lui sont associées, l'alternative entre langue et parole ne se réduisant pas à l'opposition de la partition et de l'exécution, comme chez Saussure, mais au fait que le lien entre l'une et l'autre est in fine l'instrument et les possibilités instrumentales, qu'il faut donc absolument décrire. 


\subsection{Contraintes et plurisémie} correspond), on devra d'abord remarquer (Nemo 1999) qu'il concerne la place d'un mécanisme attentionnel nommé attention contrôlée, qui consiste par exemple à prendre le regard de quelqu'un pour l'amener sur quelque chose. Or parler consiste très largement à attirer l'attention sur quelque chose en demandant à ce que cela soit pris en compte, ce qui est très précisément le propre de ce qui a été décrit par Anscombre et Ducrot sous le nom d'orientation argumentative. Dans la mesure où tout énoncé est une forme d'attention contrôlée, même si l'attention contrôlée a précédé le langage de beaucoup, il s'ensuit que tout énoncé recevra toujours une interprétation attentionnelle.

Pour que celle-ci soit pleinement argumentative ${ }^{15}$, il faut néanmoins introduire une seconde contrainte, à savoir le fait qu'attirer l'attention sur quelque chose ne peut se faire de façon neutre, autrement dit qu'il n'est pas possible de montrer quelque chose (ou de dire quelque chose) sans montrer en même temps ce que l'on pense (surprise, peur, bienveillance, etc..) ou ce que l'on souhaite que l'autre pense de quelque chose.

Dans la mesure où de surcroît le contrôle attentionnel lui-même (i.e. le fait d'attirer l'attention sur ceci plutôt que sur cela) n'est pas neutre, il s'ensuit que toute attention contrôlée est par définition manipulatoire et que toute interprétation d'un énoncé ou d'une contribution intègre obligatoirement une représentation de celui-ci comme mouvement attentionnel (et potentiellement manipulatoire) : il y a donc bien, l'attention contrôlée et le biais attentionnel qui lui est associé étant constitutif de tout acte de communication, une strate interprétative spécifique associée à toute énonciation ${ }^{16}$. Et en ce sens il n'y a tout simplement pas de sémantique linguistique possible sans strate attentionnelle, la question n'étant pas de savoir si tel ou tel sémanticien aime ou n'aime pas celle-ci, ou a envie ou non d'étudier ce qui s'y passe, mais simplement de reconnaître que la sémantique si elle veut être une science un tant soit peu exacte ne peut nier une dimension de son objet qui est constitutive de celui-ci.

Pour passer ensuite de ces contraintes attentionnelles à des contraintes énonciatives, il faut dans un premier temps remarquer que dans la mesure où parler revient à demander que quelque chose soit pris en compte, une telle demande ne sera légitime qu'à condition que ce sur quoi on attire l'attention fasse une différence : c'est ce que j'ai appelé (Nemo 1992: 137-138) ${ }^{17}$ la contrainte de scalarisation, contrainte qui est de surcroît une contrainte de pertinence.

La façon dont cette contrainte se traduit interprétativement est facile à faire comprendre, dans la mesure où :

- elle implique que tout énoncé devra répondre à la question «quelle différence cela fait-il ?»;

- la réponse à cette question, nommée valeur scalaire, est ce que la théorie de l'argumentation dans la langue a nommé « conclusion » et est donc associée à tout énoncé qui s'inscrit dans un mouvement attentionnel ;

- répondre à cette question suppose souvent de s'appuyer sur des représentations scalaires, dont la forme la plus connue sont les topoï.

S'agissant ensuite de la question de savoir si les énoncés sont associés à des biais attentionnels, autrement dit des prises de position par rapport à ce dont il est question, il est possible de constater que la question de savoir « quelle différence fait quelque chose » va dépendre très largement des alternatives introduites (et donc de l'espace de 
comparaison, autrement dit de l'image du possible ${ }^{18}$ associée à l'énoncé. Là encore, l'existence d'une strate interprétative spécifique associée au calcul de l'image du possible est non seulement prouvable mais suffisamment incontestable pour avoir été découverte plusieurs fois, puisque en dehors de mon travail, elle a donné lieu sous le nom de " sémantique des alternatives " à de très nombreux travaux qui ont progressivement confirmé, notamment chez Horn et Levinson, l'importance pour toute théorie de l'interprétation des énoncés du cadre modal qui encadre ceux-ci, l'énoncé pouvant / devant être décrit comme l'association d'une image du possible et d'une image du réel, sachant que dans l'énoncé le réel n'existe que relativement au possible.

Reste alors la dernière étape du questionnement, qui est de savoir dans quelle mesure les signes (ou d'autres contraintes) linguistiques sont à même de contraindre la construction de l'image du possible, et donc l'orientation argumentative des énoncés et enchainements et donc in fine la valeur attentionnelle de l'énonciation. Ce à quoi toute la sémantique contemporaine a contribué depuis les années soixante-dix, à la suite des travaux initiaux de Sapir (1944), en démontrant à la fois que des signes comme le couple " peu » / « un peu » étaient capables de contraindre l'orientation argumentative des énoncés mais aussi que c'était la principale fonction des connecteurs pragmatiques. Ce qui revenait à montrer que ces signes étaient porteurs d'une certaine vision du monde, dès lors que le « réel» n'existant dans l'énoncé que relativement au "possible» que celui-ci introduit (e.g. " peu vs beaucoup », « un peu vs pas du tout »), le résultat de ce type de comparaison était in fine de présenter les choses favorablement ou défavorablement.

Ce qu'il importe de comprendre enfin en outre que chacune de ces contraintes et des strates interprétatives qui leur sont associées est universelle, autrement dit s'applique à toute énonciation. Mais aussi que s'il est nécessaire de parler de strates interprétatives, et non d'étapes dans l'interprétation, c'est dans la mesure où il est empiriquement démontrable qu'il n'y a pas d'ordre fixe pour répondre à l'ensemble des questions qui se posent à tout énoncé.

\subsection{Contraintes prosodiques et points de vue}

Si le fait que l'intonation joue un rôle dans l'interprétation est pour tout un chacun un truisme, l'étude des contraintes prosodiques qui pèsent sur l'interprétation est elle toute récente en sémantique.

Parce qu'elle suppose de séparer ce qui dans la prosodie concerne ou non l'interprétation, il a été nécessaire de définir la prosodie non structurale comme «toute forme libre de réalisation intonative d'une séquence linguistique de quelque nature que ce soit à même d'exercer une contrainte sur l'interprétation (à quelque niveau que ce soit) » et ce y compris au niveau lexical où la prosodie lexicale libre est définie comme «toute forme libre de réalisation intonative d'une unité lexicale conduisant à donner à un emploi de cette unité lexicale une interprétation à la fois spécifique et réitérable » (Nemo et Petit 2015 : 86-87).

Moyennant quoi, l'étude d'un échange comme :

- Ça va?

- Ça va.

dont la structure est fixe pour ne pas dire figée, portera sur la diversité des interprétations selon l'intonation qui est associée à chacun des éléments. Tandis que 
l'étude de «enfin» ou «quelques» portera elle aussi bien sur la contribution de la prosodie à la polysémie du premier ou à l'orientation argumentative du second.

L'étude prosodique de "quelques» (Nemo, Petit et Létang à paraître) a permis de trancher une question restée jusque-là sans réponse - qui était celle de savoir si «quelques » était porteur d'une orientation argumentative et si oui de laquelle - dans la mesure où si Ducrot avait défendu la thèse d'une présentation de la quantité concernée comme significative, la plupart des dictionnaires et à leur côté nombre de sémanticiens défendaient la thèse d'une orientation inverse en glosant notamment l'interprétation de « quelques » par « peu ».

Il a ainsi été possible de montrer que selon la forme de réalisation prosodique du "quelques ", il était aussi bien possible de présenter la quantité comme significative que comme négligeable.

Ce qui pour ce qui nous intéresse ici permet de démontrer que :

- il y a bien marquage linguistique (prosodique) du jugement porté sur la quantité ;

- il n'est pas possible d'entendre les énoncés concernés sans leur associer une interprétation incluant ce jugement, ce qui signifie que l'expression prosodique du point de vue est bien une composante incontestable de la plurisémie de « quelques";

- toute représentation sémantique qui mettrait dans le mot (non intoné) les valeurs en question serait fausse, ou au mieux insuffisamment complexe.

Il s'est avéré de plus que si la prosodie était associée aux deux orientations argumentatives opposées, elle fournissait en réalité une information argumentative beaucoup plus fine à l'intérieur de chacune de ces orientations ${ }^{19}$, et permettait d'exprimer des points de vue plus complexes, en particulier en étant capable de mettre en scène une pluralité de points de vue se répondant (et donc de la polyphonie).

Il semble donc clair que la sémantique de la prosodie est en passe de fournir à la thèse de l'inscription linguistique des points de vue portée par Pierre-Yves Raccah les preuves incontestables dont elle avait besoin. Ceci dans la mesure où le caractère indubitablement linguistique de la prosodie, ainsi que le caractère universel de la prosodie, va rendre intenable la thèse inverse, les tenants de celle-ci se retrouvant à devoir définir soit le caractère non-linguistique de la prosodie soit que l'intonation n'est pas porteuse de points de vue.

En prouvant l'existence d'un profilage prosodique de l'orientation argumentative mais aussi la très grande diversité des commentaires prosodiques qui peuvent être associés à un même signe, l'étude de la prosodie va par ailleurs libérer la sémantique des points de vue de la nécessité de démontrer que ceux-ci sont codés dans le morphème : il deviendra donc possible de démontrer que l'argumentation est bien dans la langue ou encore que des points de vue sont bien associés aux emplois des signes sans avoir pour autant à démontrer que ceux-ci sont codés par le morphème.

De nouveaux chantiers sont en revanche à prévoir, dès lors qu'en intégrant la dimension prosodique, la sémantique des points de vue va devoir se confronter à des phénomènes à certains égards plus complexes que ce qu'elle avait pu mettre à jour jusqu'ici.

Le premier problème (Létang et Nemo à paraître) concerne la représentation des orientations argumentatives dans les enchaînements.

Si l'on prend en effet un enchaînement de type :

il a lu quelques livres mais il y a longtemps 
51 qui est traditionnellement décrit comme opposant deux énoncés anti-orientés $p$ et $q$ (menant à des conclusions opposées $r$ et non-r) et que l'on prend en compte la prosodie (et notamment la réalisation prosodique de "quelques»), on constate que le contour prosodique associé au premier énoncé est réalisé dans beaucoup de cas avec une présentation minimisante de la quantité en question. Ce qui implique que l'énoncé p est prosodiquement co-orienté avec q et présente prosodiquement la quantité mentionnée comme faible et peu significative. Ceci alors que "mais» quant à lui continue de présenter les deux énoncés comme menant à des conclusions opposées.

52 Si la cause d'un tel phénomène est identifiable, dès lors que " $p$ mais $q$ " n'est pas seulement un enchaînement d'énoncés (avec des orientations argumentatives pour chacun de ceux-ci) mais une contribution [pq] à laquelle une valeur argumentative globale est attachée, le commentaire prosodique minimisant associé à $\mathrm{p}$ et dans $\mathrm{p}$ à "quelques» devant être interprété comme une anticipation de l'orientation de la contribution dans son ensemble, il reste que le simple fait que le segment p puisse être porteur de plusieurs niveaux d'orientations argumentatives va impliquer pour toute sémantique des points de vue de complexifier quelque peu l'appareil descriptif existant et notamment de modéliser le rapport entre la plurisémie générée par chaque niveau de contraintes et la polyphonie qui en résulte au niveau d'un énoncé donné, comme ici le segment $\mathrm{p}$.

53 Le second problème et chantier à venir concerne le rôle de la prosodie dans le profilage des sens lexicaux et sa place dans la représentation des mots. Il s'avère en effet, comme je l'ai montré avec Mélanie Petit à propos de enfin qu'il est indispensable pour représenter correctement le lexique d'adopter une représentation dualiste du signifiant et du sens dit lexical faute notamment de pouvoir associer les interprétations lexicalisées avec la seule forme phonématique du mot.

Ce qui conduit à définir les emplois-types comme les véritables unités lexicales ultimes d'une langue et d'en faire la représentation suivante (Nemo et Petit 2012 : XX1)

Emploi-type :

Phon : $\sigma \varphi$ : valeur (forme phonématique)

$\pi$ : valeur (forme prosodique)

Sem : $s \psi$ : valeur (interprétation-type)

$\rho$ : valeur (commentaire argumentatif)

60 Statut grammatical $\gamma:$ valeur

61 Représentation plurisémique qui repose sur l'existence d'une strate de signification / indication morphémique ${ }^{20}$ (partagée par tous les emplois du mot), d'une strate de profilage sémantique (interprétation-type ${ }^{21}$ ) et d'une strate de profilage prosodique (emploi-type ${ }^{22}$ ).

Une telle évolution a évidemment des conséquences importantes pour toute sémantique des points de vue aussi bien pour la question de savoir où commence la lexicalisation des interprétations que pour celle de savoir si les points de vue sont associés aux emploistypes, aux interprétations-types ou à la signification morphémique, sachant qu'il n'est pas possible de préjuger que ce « ou » doive être un « ou » exclusif.

63 La troisième observation concerne le fait que la prosodie dans certains cas au moins ne semble pas exprimer un rapport avec ce qui est dit mais plutôt un rapport avec ce dont on 
parle indépendamment de ce qu'on en dit, autrement dit être en quelque sorte en position thématique, comme c'est le cas par exemple d'un énoncé comme l'énoncé « il n'y a pas mort d'homme", auquel est conventionnellement associé (y compris prosodiquement) le point de vue «ce n'est pas grave», et ce alors que le «ce» qu'il comporte ne porte pas sur «il n'y a pas mort d'homme », puisqu'il n'est pas dit du fait qu'il n'y a pas mort d'homme que ce n'est pas grave, mais exactement l'inverse à savoir «ce n'est pas grave puisqu'il n'y a pas mort d'hommes ». Le tout impliquant que ce qui est dit peut parfaitement commenter ce que dit la prosodie là où on s'attendrait à ce que l'inverse aille de soi.

64 Ces trois problèmes et beaucoup d'autres font qu'il sera très important de clarifier à l'avenir la relation entre construction prosodique et non-prosodique des points de vue et par exemple de clarifier le rôle respectif de "quelques », de «mais » et de la prosodie associée à « quelques » dans l'identification des points de vue associés à un enchaînement et du rapport de l'énonciateur à ceux-ci - et qu'il ne fait donc guère de doute que la sémantique des points de vue deviendra aussi une sémantique de la prosodie.

\section{Sémantique des points de vue et sémantique de la référence}

65 Jusqu'ici, je me suis contenté de rendre explicites toutes les raisons qui font que postuler l'existence d'une strate interprétative associée aux points de vue et biais attentionnels est pour toute théorie sémantique indispensable.

À certains égards cependant, des distinctions comme la distinction entre dénotation et connotation ont toujours permis de reconnaître qu'il y avait plus dans le sens lexical que la dénotation. Ce qui n'empêchait pas de considérer ces strates comme secondaires ou localisées à certains types de mots. Position que certains continuent de maintenir en concédant que les entrées lexicales sont complexes mais en défendant l'idée que le sens lexical est lui atomique et qu'il s'identifie avec la dénotation :

Finally, we defend a sort of lexical atomism: though, strictly speaking, we concede that lexical entries are typically complex, still we claim that their complexity does not jeopardize either the thesis that lexical meaning is atomistic or the identification of lexical meaning with denotation. (Fodor et Lepore $1998: 269$ )

Ce à quoi je vais consacrer le reste de ce travail va être de tenter de montrer au contraire qu'une des traces repérables de points de vue dans les énoncés s'observe dans la strate dénotationnelle et référentielle.

La sémantique, comme beaucoup sinon la plupart des disciplines scientifiques n'accède à ses objets qu'en tant que ceux-ci laissent des traces qui seraient autrement inexpliquées. La position que je vais donc être amené à défendre est que la notion de point de vue joue ainsi un rôle analogue à celui que joue une hypothèse comme celle de la courbure de l'espace-temps en physique, à savoir de rendre compréhensibles des observations qui sans cela seraient incompréhensibles.

69 Le propre des contraintes interprétatives comme celle qui résulte du fait que l'attention contrôlée soit constitutive du langage (et en conséquence des langues), est de laisser toute sorte de traces ${ }^{23}$ qui sont en réalité la seule forme de preuve dont le sémanticien dispose. Pierre-Yves Raccah $(2002,2006)$ a souvent eu l'occasion de le dire, notamment quand il souligne que «ni les phrases, ni la signification, ni même le sens ne sont des 
entités observables » et qu'un subterfuge est alors «l'observation indirecte, c'est-à-dire l'observation de phénomènes accessibles aux sens, dont on fait l'hypothèse qu'ils ont été causés par le phénomène que l'on veut décrire et qui, lui, n'est pas directement accessible aux sens » (Raccah $2006: 124)$.

Ce que je vais tenter de montrer à l'aide d'un ensemble d'exemples, c'est donc la façon dont des phénomènes interprétatifs parfaitement objectivables, et relatifs précisément à l'interprétation dénotationnelle-référentielle-vériconditionnelle ${ }^{24}$, s'avèrent être la trace de l'existence de points de vue / rapport aux mondes / idéologies que les interprétants peuvent et doivent reconstruire.

Il s'agira en particulier de montrer que la distinction entre extension et intension, et le fait que l'extension comme la dénotation dépende de l'intension, permet d'observer des phénomènes qui se situent aux antipodes de l'idée que les phrases combineraient des items lexicaux dont le contenu sémantique principal, fixé lexicalement, serait dénotatif.

\subsection{Points de vue et sémantique comparée}

72 Mon point de départ concernera néanmoins une question conçue habituellement comme relevant de la sémantique comparée, mais qui illustre en réalité le même problème que celui qui se pose quand on doit interpréter ce à quoi renvoie telle ou telle partie d'un énoncé ordinaire.

Imaginons en effet que je découvre qu'une langue amérindienne comme le Palikur utilise le même terme auna pour ce qui serait en français « (des hommes qui) parlent » et « (des animaux qui) vocalisent », ce qui conduit habituellement à dire que ces deux langues " découpent » le monde de façon différente, ou encore qu'elles posent comme continue(s) (ou discontinue(s)) telle(s) ou telle(s) réalité(s), à charge ensuite de savoir si elles ont deux visions du monde différentes (thèse relativiste) ou si elles ont juste deux variantes d'une vision commune à tous les humains (thèse universaliste).

74 La réalité est pourtant plus complexe, et à bien des égards plus intéressante. Pour commencer dans la mesure où il n'est par exemple pas possible de dire que cette langue emploie le verbe "parler » indistinctement pour humains et non-humains, puisque cela reviendrait à projeter le sens français de "parler » sur la langue en question. Et ensuite parce qu'il n'est pas nécessaire pour autant d'en conclure que chaque langue serait incommensurable à l'autre.

Ce qui compte en effet se situe ailleurs, à savoir dans le fait que tout emploi du même terme pour les deux réalités concernées (de notre point de vue) va obligatoirement conduire à devoir sélectionner dans chacune ce qui la rapproche de l'autre, autrement dit va fonctionner comme une instruction. Et que l'inverse se produira en cas d'emploi de deux termes distincts. Ce qui dans les deux cas, pour des raisons que nous allons étudier, forcera soit à prêter attention à ce qui différencie là soit à prêter attention à ce qui est semblable.

Le problème n'est donc pas que les deux langues « découpent » le monde différemment mais qu'elles obligent dans chaque réalité à voir quelque chose comme semblable ou comme différent en focalisant l'attention sur ce qui est semblable ou sur ce qui est différent ${ }^{25}$. 


\subsection{Sélection pertinentielle de la référence des termes par les prédicats} largement en sens inverse et que ce sont les prédicats qui vont en quelque sorte sélectionner et donc définir et l'extension d'un terme et ce qu'il faut appeler son « intension en emploi ». représentée comme :

Il existe un prédicat $\mathrm{P}$ qui pour qu'il soit applicable à / vrai de $\mathrm{X}$ et $\mathrm{Y}$ force à admettre que $X$ et $Y$ soient $X^{\prime}$ et $Y^{\prime}$. 
Ce qui par ailleurs est tout à fait remarquable, tant linguistiquement que philosophiquement, est de surcroît que comme dans la négation d'un énoncé, il en est exactement de même, il s'ensuivra, contre toute attente, que l'énoncé négatif ne niera pas le plus souvent la même chose que ce qui est présent dans l'énoncé positif ${ }^{29}$.

Ce qui revient à dire que si je voulais en effet défendre la thèse selon laquelle « le langage n'est pas le propre de l'homme», je serai en effet amené à devoir défendre une conception du sens de «langage » qui ne serait pas la même que celle qui serait défendue par le tenant de la version affirmative de l'énoncé. Ce qui en termes logiques, impliquerait de dire que là où l'énoncé sous sa forme affirmative dit quelque chose comme :

« Il existe L' et H' tel que $\mathrm{P}\left(\mathrm{L}^{\prime}, \mathrm{H}^{\prime}\right)$ est vrai »

l'énoncé sous sa forme négative dira lui quelque chose comme :

« Il existe L" et H" tel que non-P(L", H") est vrai »

avec donc un L" différent de L' et un H" différent de H'.

Forme dont il est possible de montrer qu'elle est la traduction logique du problème évoqué à propos de " parler », à savoir que les conditions de vérité ou d'applicabilité sont liées à des points de vue.

\subsection{Prédications analytiques}

Plus étonnant encore, on retrouvera cette antériorité / primauté de la prédication dans l'interprétation des propositions dites analytiques, définies comme les propositions qui sont vraies en vertu de leur seule signification (ou bien en vertu de leur définition).

Prenons en effet l'exemple traditionnel, "bachelors are unmarried men ", dont la (problématique) traduction en français est «les célibataires sont des hommes nonmariés ", à propos desquels on a donc admis en somme que " unmarried men » serait la signification / définition de «bachelors » ce qui rendrait la prédication analytiquement vraie.

Or que constate-t-on en réalité ?

Que les mots célibataires (en français) et bachelors en anglais :

- sont employés pour d'autres intentés que des unmarried men (par exemple tout à fait couramment en zoologie et en éthologie), certains n'étant pas des humains, d'autres n'étant pas des humains mâles (en ce qui concerne l'anglais ${ }^{30}$ );

- ne sont pas employés pour les mineurs, ce qui implique que men est interprétés comme « hommes adultes";

- ne sont pas employés pour des hommes qui ne vivent pas seuls mais ne sont pas mariés pour autant $t^{31}$, par exemple des hommes vivant en concubinage ;

- ne seront pas employables dans certains contextes (notamment administratifs) à propos de gens qui ont été mariés mais ne le sont plus (divorcés, veufs) ;

- seront employables à propos d'enfants dans les mêmes contextes.

Ce qui dans tous les cas montre que :

- l'emploi de ces termes n'est aucunement contraint par le prédicat présenté comme analytique ;

- le fait de ne pas vivre en couple (ou harem, etc.) alors qu'on serait en âge le faire semble être la contrainte partagée par tous les emplois ;

- dire de ce prédicat qu'il serait le sens lexical ou la signification n'a pas beaucoup de sens, sauf à considérer que la signification ou le sens lexical ne détermine en rien 
la façon dont les mots sont employés ;

- chaque élément du prédicat est lui-même amené, si la proposition énoncée doit pouvoir devenir vraie, à devoir être associé à une interprétation particulière ;

- la langue n'a aucun pouvoir de définition des normes sociales ou comportementales qui régissent la vie de couple (ou équivalent) ;

- la société a le cas échéant le pouvoir de définir celles-ci, y compris de définir comme célibataires des concubins ayant des enfants mais en ce cas, il s'agira de célibataires qui ne le sont que de nom, et il ne s'agira pas d'appliquer un sens lexical sans point de vue mais de promouvoir une façon de voir la réalité (l'intenté) consistant à ne pas la voir ;

- le sens lexical ou la signification d'un terme ne sont tout simplement pas des définitions de ce à quoi il s'applique ;

- un terme n'a pas UNE intension et UNE extension.

Le tout impliquant que l'interprétation n'est pas un processus d'association de sens lexicaux mais un processus de satisfaction de contraintes. Dont la principale est de trouver quelque chose qui puisse rendre vrai ce qui est prédiqué en adaptant l'interprétation de ce à propos de quoi la prédication est énoncée. Comme en témoigne éloquemment le fait que les exemples cités aient pu aussi longtemps servir d'exemples d'analyticité.

\subsection{Conflits référentiels}

99 S'il est tout à fait rare que ce principe ne soit pas appliqué, la situation se produit néanmoins parfois.

100 Un exemple en est le débat sur le changement ou non de millénaire en l'an 2000, où certains ont tenté de convaincre que fêter le changement de millénaire au premier janvier était une énorme illusion collective, celui-ci ne devant se produire qu'au $1^{\mathrm{er}}$ janvier 2001. Moyennant quoi il était faux et inacceptable d'employer l'expression en l'an 2000.

Il est clair pourtant que l'emploi de l'expression avait en réalité pour intenté le «changement de chiffre des milliers au $1^{\text {er }}$ janvier 2000 » et que ce changement étant incontestable, et étant précisément l'objet de l'excitation collective, les habitants de la planète avaient une bonne raison de ne pas attendre le $1^{\text {er }}$ janvier 2001 pour fêter le changement de millénaire. Moyennant quoi le sémanticien est confronté à deux "découpages » du temps : l'un qui identifie un millénaire avec la période qui s'écoule de l'an 1000 à l'an 1999 (ou de toute autre fourchette d'un millier d'années), l'autre qui identifie nos millénaires avec des périodes allant de toute année N001 à l'année $\mathrm{N}^{+1} 000$, par exemple de l'an 1001 à l'an 2000. La raison de ce décalage est en l'espèce facile à identifier, même si la quasi-totalité des gens l'ignorent, à savoir l'absence d'année 0 , qui conduirait en appliquant la logique du premier point de vue à avoir un premier millénaire de 999 ans.

Le problème du sémanticien n'est pas bien évidemment de choisir entre Charybde et Scylla. Il se contentera de noter la co-existence de deux points de vue distincts, dont l'un fait « culturellement» autorité mais heurte les patterns interprétatifs et les conventions langagières standards. Et donc de relever :

- l'existence d'une interprétation ordinaire (et massivement partagée) en vertu de laquelle le descripteur « changement de millénaire » est applicable si changement il y a dans les dates en ce qui concerne le chiffre des milliers ;

- le fait que cette interprétation ordinaire satisfait l'exigeante définition du sens de 
Bloomfield ;

- le fait que cette interprétation satisfait aussi le caractère de fait social total u qui définit la langue depuis Saussure dès lors que personne n'ira critiquer Victor Hugo pour avoir écrit « ce siècle avait deux ans » en parlant de l'année 1802 ou contester le fait que l'Italie dans les années trente, ait pu célébrer les trois bimillénaires de la naissance de Virgile en 1930 (né en moins 70), Horace en 1935 (né en moins 65), Auguste en 1937 (né en moins 63) ;

- l'existence d'une définition savante et normative en vertu de laquelle l'absence d'année zéro faisant que la fin de la première année était la fin de l'an un, et un millénaire ne pouvant commencer que si le précédent est fini, il faut reporter ce décalage et considérer que tout nouveau siècle ou millénaire aura une date avec 1 comme chiffre des années ;

- le fait que le changement de chiffres des années se produisant tous les ans est beaucoup moins rare que le changement de chiffres des siècles qui n'arrive presque jamais deux fois dans la vie d'un homme, et a fortiori qu'un changement du chiffre des millénaires que la plupart des humains ne vivent jamais ;

- le fait que plus un moment est rare plus il mérite d'être célébré ;

- le fait que le prédicat «mérite d'être célébré » sera donc vrai pour l'an 2000 et faux pour l'an 2001 ;

- le fait que quand il y a "changement de millénaire dans la date» il y a interprétativement « changement de millénaire »;

- le fait que la notion de millénaire s'applique à toute durée de mille ans quelque soit son point de départ ;

- le fait qu'employer début du troisième millénaire pour le $1^{\text {er }}$ janvier 2000 implique donc de définir le second millénaire comme allant de l'an 1000 à l'an 1999 ;

- le fait que l'appartenance de l'année 1000 à l'ensemble des années commençant par 1000 est une évidence tant verbale (il faut dire mil) que graphique ;

- le fait que l'ignorance de l'absence d'années zéro dans notre calendrier est généralisée ;

- le fait que les sujets parlants peuvent comprendre que faute d'année zéro le changement de date n'est pas synonyme d'écoulement complet de deux millénaires mais synonyme de continuer fêter ce qui compte pour eux à savoir un changement radical de la date.

$103 \mathrm{Au}$ total, on voit que le fond du problème concerne la relation entre le prédicat « célébrer » et l'interprétation de « millénaire ${ }^{32}$, sachant que :

- l'énoncé «il n'y a rien de particulier à célébrer en l'an 2000 » est faux (i.e. a été

falsifié par les intéressés);

- ce qu'il y a à célébrer est bien, en un certain sens, un changement de millénaire ;

et que le grippage du processus de sélection de la référence par le prédicat tient sémantiquement à ce que l'interprétation la plus "analytique » s'est heurtée à une définition qui ne l'est pas du tout mais qui elle aussi a sa part de vérité. Quiconque aurait eu le sens du compromis aurait pu dire «le $1^{\mathrm{er}}$ janvier 2000 ne sera pas un vrai changement de millénaire puisque le premier millénaire n'a duré que 999 ans ", énoncé qui mélange allégrement les points de vue tout en étant parfaitement compréhensible.

\subsection{Points de vue et détermination de la référence}

Quand un syndicaliste policier dit «ce sont des armes qui peuvent être utilisées contre des policiers, ou même contre des français » la question se pose peu de savoir s'il y a expression d'un point de vue ou non ${ }^{33}$, sachant qu'en tout état de cause, on peut observer que l'extension du descripteur «des français » est transformée par son cotexte pour se réduire à « les français qui ne sont pas policiers ». 

résulte. l'Amérique.

Or, cette transformation incontestable d'un ensemble en un sous-ensemble de lui-même par promotion d'un de ses sous-ensembles en ensemble alternatif ne peut être ni expliquée ni décrite dans le cadre d'une description dénotationnelle-vériconditionnelle réduite à un ensemblisme statique, étant la résultante du fait que les formes de satisfaction des contraintes sémantiques introduites par chaque élément dépendent du pool de contraintes qui est introduit en emploi et du système de contraintes qui en

Une telle réalité n'est il faut le noter aucunement liée à cet exemple particulier qui vient d'être, ou attribuable à la maladresse d'un syndicaliste, puisqu'on observe exactement le même phénomène dans des contextes où la création en emploi d'une opposition entre deux termes qui a priori n'ont pas de raison de l'être passera totalement inaperçue.

L'exemple que je vais utiliser est celui de la question du peuplement des Amériques, où il s'agit initialement de répondre à la question de savoir qui est arrivé en premier et à quel moment, question qui déchaîne les passions depuis cinquante ans.

Pour le rendre intelligible, je commencerai par résumer ce qu'une réponse directe à la question pourrait être avant de voir comment une autre présentation des choses est habituellement adoptée.

Une telle réponse serait la suivante :

- la première partie des Amériques à avoir été peuplée a été une zone allant jusqu'au sud de l'Alaska et qui faisait partie d'une zone nommée Béringie qui s'étendait des deux côtés de ce qui est aujourd'hui le détroit de Béring mais qui à l'époque était émergée ;

- on ne sait pas exactement à quel moment mais la présence en Béringie a pu précéder de 10000 à 24000 ans la dispersion sur l'ensemble des Amériques ;

- celle-ci a eu lieu au plus tôt vers $16000 \mathrm{AP}^{34}$, la présence en Amérique du sud étant elle admise à partir de $14800 \mathrm{AP}$.

L'intenté étant maintenant fixé, nous pouvons voir maintenant comment va être employé le terme « les Amériques » dans la littérature sur le sujet.

Ce qui frappe en effet dans celle-ci est que :

- nulle part n'est jamais dit que le continent américain n'a pendant longtemps été occupé que partiellement ;

- il n'est jamais fait mention explicite de l'Amérique quand il s'agit d'évoquer le peuplement de l'Alaska et du nord de la Colombie britannique. Comme si l'Alaska n'était pas en Amérique et était encore moins l'Amérique ;

- le terme «les Amériques » n'est très vite employé que pour désigner une zone se situant au sud de la calotte glaciaire qui barrait alors le continent d'Est en Ouest au sud de la Béringie, Comme les Amériques ne commençaient qu'au sud de cette calotte et comme si une nouvelle fois ce qui était au nord n'était pas déjà

L'emploi récurrent de l'expression « into the Americas » est en effet réservé au passage au sud de cette cellote et associé à l'expression «dispersal into ${ }^{35}$ the Americas » qui doit s'interpréter comme "dispersion à partir du Nord-ouest vers le reste des Amériques " mais qui, à la manière de l'opposition " policiers » / " français » de l'exemple précédent, s'inscrit dans une opposition entre « Béringie » et " Amériques ».

Or une telle restriction, quelle qu'en soit la cause (et plusieurs hypothèses peuvent être avancées à ce sujet), n'a pas d'autre fondement qu'elle-même : les continents ne sont en général pas définis par la distribution des masses glaciaires et la création d'une opposition entre Béringie et Amériques à l'intérieur de laquelle la Béringie devient une 
non-Amérique revient une nouvelle fois à définir un ensemble par un de ses sousensembles en transformant un autre sous-ensemble en ensemble alternatif. Ce qui dans l'absolu n'est pas plus fondé que d'avoir l'air de considérer les policiers comme ne faisant pas partie des français.

Ce qui importe pourtant est que les lecteurs ne s'y perdent pas et que la restriction en question, loin d'être un problème, va surtout devoir être interprétée, et qu'ils seront donc conduits :

- à suivre les chercheurs dans leur obsession constante pour le passage de la calotte glaciaire et le peuplement de la vraie Amérique, celle qui commence au sud du Canada ;

- à comprendre les réticences de ceux-ci à commencer par le plus incertain (quitte à ne pas répondre à la question) et leur propension à vouloir parler de ce qu'ils comprennent le mieux ou le moins mal (la fameuse dispersion);

- à reconnaitre dans les formulations choisies les traces du dogme qui a longtemps interdit sous peine de disqualification le fait d'avancer quoi que ce soit sur une arrivée pré-Clovis (avant moins 12000 ans AP) dans les Amériques.

En tous cas, on voit se stabiliser une conventionalisation de la restriction et de l'utilisation de la calotte glaciaire infranchissable comme critère intensionnel :

One model based on Native American mtDNA Haplotypes (Figure 2) proposes that migration into Beringia occurred between $30 \mathrm{k}$ and $25 \mathrm{k}$ cal years $\mathrm{BP}$, with migration into the Americas occurring around $10 \mathrm{k}$ to $15 \mathrm{k}$ years after isolation of the small founding population ${ }^{36}$.

\subsection{Restriction référencielle et sémantique}

Reconnaître l'existence des phénomènes de réduction de la portée d'un terme que je propose d'appeler « restriction sémantique » interdit de postuler comme c'est le cas dans toutes les sémantiques logiques et formelles (e.g. le lambda-calcul de Montague) que l'application d'un prédicat présuppose l'existence d'arguments de celui-ci qui seraient définis indépendamment du contenu prédiqué et que sa négation peut imposer comme nous l'avons vu des restrictions sémantiques distinctes de celle du prédicat non-nié.

Comprendre ensuite que ces restrictions sémantiques sont les traces d'une présomption de pertinence, autrement dit du fait que ce qui est dit est toujours présumé être « d'une certaine façon» vrai, permet de comprendre que c'est précisément dans la nécessité d'ajouter ce "d'une certaine façon» ou "dans un certain sens» que réside la strate associée à ce qui a déjà été mis à jour sous le nom de « points de vue ».

Sur cette base, il est alors possible de comprendre que ces variations sémantiques et leur caractère naturel supposent :

- la capacité d'adopter ou de faire sienne une certaine façon de voir les choses, qui est constitutive de l'empathie et de ce que les psychologues appellent attention conjointe et attention déictique (Conein 1998);

- que la première chose que quelqu'un verra quand il suivra le regard de quelqu'un sur quelque chose (attention conjointe) sera le regard lui-même, moyennant quoi ce à quoi ce regard donnera accès dans le monde ne sera jamais saisi indépendamment de la nature du regard porté.

S'explique alors que la non-transparence du vecteur attentionnel, autrement dit du moyen ${ }^{37}$ spécifique qui est concrètement utilisé pour aller chercher l'attention de quelqu'un et l'amener sur quelque chose est ce qui rend obligatoire l'existence d'une strate interprétative associée à l'interprétation de ce vecteur. 


\subsection{Points de vue et interprétation des expressions de la quantité} vrai qu'ils sont des passagers. Ce qui, même en admettant que l'ensemble en question soit limité aux personnes présentes au moment de l'énonciation, inclut les enfants. Et il en serait de même pour chaque autre signe, avec «des" renvoyant à l'ensemble des individus ayant au moins deux enfants, avec «enfant» renvoyant à l'ensemble des individus dont il est vrai qu'ils sont « enfants " y compris si cela inclut ceux qui vérifient la propriété « $\mathrm{X}$ est enfant de $\mathrm{Y}$ » (autrement dit tout le monde), etc. Ce qui au total conduirait à l'interprétation un peu baroque que j'ai essayé de formuler, qui a au moins le très grand intérêt heuristique de montrer que croire que « le sens d'une phrase est ce qui la rend vraie » ne doit pas être compris comme une évidence axiomatique mais comme un immense travail pour l'interprétant, qui doit constamment imaginer une forme de vérité qui soit un tant soit peu censée.

Le décalage entre les deux interprétations concerne notamment :

- l'interprétation du un déjà signalé ;

- le fait de se présenter ou non avec leurs / des enfants

- le fait que s'ils sont avec leurs enfants mais que ceux-ci sont adultes alors ces 
derniers ne sont pas considérés comme des enfants ${ }^{38}$;

- le fait que les passagers enfants qui voyagent avec d'autres enfants ne sont pas les destinataires de l'énoncé ni les passagers auxquels il est demandé de se présenter.

Ce qui à chaque fois interdit de supposer que chacun des termes puisse être défini en amont de l'assertion du prédicat.

On voit donc que l'interprétation, loin de supposer que "passagers » par exemple doit référer à l'ensemble des $\mathrm{X}$ qui sont (sur la liste) des passagers (enregistrés) et à propos de qui il sera ensuite dit quelque chose de vrai, fonctionne en réalité en sens inverse. Et qu'il faudra d'abord comprendre pourquoi l'énoncé est énoncé (autrement dit la différence que peut faire dans le contexte d'être accompagné d'enfants ou non) pour comprendre ensuite que tous ceux qui sont accompagnés de personnes qui satisfont telle ou telle des propriétés évoquées mais qui ne sont pas pertinentes du point de vue de l'objectif visé dans la situation d'énonciation vont devoir être exclus de la liste des référents visés.

Savoir qui exactement est visé dans l'emploi d'un terme suppose donc de comprendre préalablement l'ensemble de l'énoncé (notamment d'un point de vue pragmatique). Ce qui, pour paraphraser le principe de Frege selon lequel les mots n'ont de sens que dans la phrase où ils sont employés, revient à dire que « les phrases (et donc les mots qu'elles comportent) n'ont d'interprétation que dans les énoncés où elles sont employées » et qu'elles ne transmettent pas de sens mais seulement des contraintes à satisfaire.

131 Ainsi est-il possible de prouver que le prédicat "avoir trois ans", s'il apporte des contraintes à satisfaire, n'a pas d'interprétation linguistique indépendante du contexte d'énonciation, et n'aura d'interprétation dans celui-ci que pour autant qu'il sera associé à une façon particulière de voir les choses.

Prenons en effet les exemples suivants :

(1) Je n'ai pas 3 ans mais 3 ans et demi (dit par un enfant)

(2) Il n'a pas 3 ans mais 3 ans et demi (dit par un(e) spécialiste de l'acquisition du

langage menant une enquête)

(3) Il n'a pas 3 ans et il sait déjà écrire quelques lettres.

(4) Il n'a pas 3 ans mais 5.

\section{Que constatons-nous?}

D'abord que ce qui rend vrai « ne pas avoir 3 ans » diffère dans les quatre cas et qu'il n'existe aucun sens phrastique qui - si l'on suppose qu'il est transmis à toute énonciation de la phrase - puisse faire partie du sens du prédicat dans chacun des énoncés. Ce qui revient à dire qu'il n'y a pas de strate interprétative d'interprétation de la phrase préalable à l'interprétation de l'énoncé.

Ensuite que la principale différence entre les interprétations qui partagent l'interprétation-type « il a plus de 3 ans » est de savoir si l'on compte en années, en demiannées ou en mois. Moyennant quoi, la question de savoir par exemple si « avoir 3 ans et demi » est un sous-ensemble "d'avoir 3 ans » ou un ensemble alternatif dépendra uniquement de la question de savoir si de telles différences comptent ou ne comptent pas dans le contexte d'emploi.

Sachant qu'il n'y a en réalité pas de réponse absolue à cette question qui puisse devenir une norme linguistique contraignante et être autre chose qu'une simple habitude dont la langue peut librement s'affranchir. La réponse selon laquelle il faudrait compter en années ne consiste en effet qu'à poser que les différences inférieures à l'année ne comptent pas, ce qui : 
- ne sera pas toujours le cas comme le montrent mes deux premiers exemples;

- restent comme toute affirmation relative à ce qui compte ou ce qui ne compte pas une pure question de point de vue.

\section{La question de la lexicalisation des points de vue et de la signification}

Reste au terme de ce périple à revenir à la question qui a été au cœur du travail de PierreYves Raccah à savoir celle de l'inscription en langue des points de vue.

Cette question peut être abordée à la fois de façon globale, en termes d'inscription grammaticale (e.g. la grande masse de travaux menés sur la sémantique des temps et des modes) et en termes d'inscription dans le lexique.

Or, répondre à cette dernière question conduit en réalité à devoir se demander où commence exactement le lexique mais aussi où commence et s'arrête la signification, sachant que ces deux interrogations concernent au bout du compte la conception qu'une théorie sémantique, en tant que telle, doit avoir du lexique, et impliquent comme nous allons le voir de rompre avec la façon dont celui-ci est représenté ou conçu dans les dictionnaires.

Pour des raisons de contrainte de taille des entrées comme de conception intrinsèque du lexique, les représentations lexicographiques tendent en effet :

- à laisser penser que la strate dénominative est première et donc la question "que veut dire $\mathrm{X}$ ?» et «qu'est-ce qu'un $\mathrm{X}$ ? » sont synonymes, la réponse à la seconde question valant réponse à la première ;

- à négliger pour le coup l'existence de beaucoup d'emplois non-dénominatifs et le caractère totalement lexicalisé de ceux-ci ;

- à ne décrire plusieurs dimensions sémantiques de l'interprétation des mots concernés qu'incidemment et en quelque sorte clandestinement;

- à ne pas le plus souvent décrire en tant que telles les divergences qui peuvent exister quand aux conditions d'applicabilité d'un mot et à choisir de privilégier celles qui au moment de la rédaction de l'article font soit autorité soit consensus.

Or, s'agissant ensuite d'une sémantique du lexique, la question n'est pas tant de savoir comment gérer le fait de ne disposer que d'un espace limité pour décrire les emplois d'un mot en se concentrant sur les emplois considérés comme les plus importants, mais celle de savoir à quel moment il est possible et nécessaire de parler de lexicalisation d'un emploi. Autrement dit, de savoir si on a affaire à une interprétation ponctuelle, et qui va donc disparaître juste après avoir été obtenue, ou si cette interprétation va se lexicaliser.

Les réponses de base à cette question sont les suivantes :

- une interprétation n'est nouvelle que si elle n'a jamais été obtenue antérieurement ;

- dès qu'un emploi va se répéter, l'interprétation qui lui est associée va devenir, si elle a pu être mémorisée, disponible sans « recalcul »;

- c'est précisément à ce processus de mémorisation des interprétations associées aux emplois que correspond la notion de lexicalisation et l'idée d'une disponibilité durable dans le lexique (par «stockage» des interprétations obtenues) des sens d'un terme ;

- toute transformation d'un emploi en usage conduit à une lexicalisation ;

- le fait pour un emploi de devenir dénominatif en fait un usage ;

- un emploi non-dénominatif peut parfaitement devenir un usage et se conventionaliser. 

fera partie. sémantique. quelques jalons.

Or, une telle définition des conditions de lexicalisation implique que devra être inclu dans le lexique l'ensemble des emplois-types d'une unité, et qu'il faudra admettre que tout emploi récurrent dont l'interprétation est partagée ${ }^{39}$ par la communauté pertinente en

La sémantique des points de vue, par exemple les travaux récents de Laetitia Pille (2015), est donc fondée à considérer que ce que l'on pourrait nommer les « acceptions » d'un mot font pleinement partie du lexique et que la diversité de ces acceptions relève d'une forme omniprésente de polysémie qui mérite beaucoup plus d'attention.

On peut de même s'appuyer sur la sémantique de la prosodie pour constater que les paires formes / sens prosodiquement pertinentes lexicalement sont elles-aussi caractérisables comme des emplois-types. Ce qui concrètement signifie que deux interprétations argumentativement distinctes d'un mot comme "minimiser ", à savoir « faire savoir que les conséquences sont / seront minimes » et " prétendre indûment que les conséquences sont / seront minimes » (Nemo 2014) seront très largement prédictibles prosodiquement, ce qui implique que lexicalement il n'y a en réalité pas d'ambiguïté

Tout ceci revient à dire que si les points de vue associés aux acceptions ou aux paires forme phonématique / forme prosodique sont obligatoires pour toute sémantique du lexique, c'est en tant que le lexique effectif des langues ne peut être identifié avec la version réduite (car simplifiée et parcellaire) que les meilleurs dictionnaires - sans parler des représentations formelles - peuvent en donner. Ceci en particulier parce que la logique de profilage de l'interprétation dans les emplois ne peut se décrire comme une liste de sens et doit l'être minimalement comme une arborescence interprétative rendue obligatoire par la plurisémie.

7 Si donc la lexicalisation des points de vue associés aux interprétations des emplois devenant des usages ne fait aucun doute une fois admis que le lexique est sémantiquement très différent de ce que le sens commun (fut-il savant) nomme sous ce nom, reste ensuite la question de savoir jusqu'à quel point la double pertinence des notions de signification linguistique et de points de vue doit conduire à concevoir la première comme portant inévitablement sur les seconds.

Dans ce débat, qui mériterait un article à lui tout seul, je me contenterai de poser

Le premier est que toute conception bisémique de la plurisémie, comme la distinction signification / sens, conduit à vouloir mettre dans la signification toute forme de stabilisation de l'interprétation, ce qui rend empiriquement fragile les théories concernées, exposées à être falsifiée, même quand $99 \%$ des feuilles relèvent d'une seule branche, par l'existence du $1 \%$ restant. Et ce qui peut conduire à ce que soient au bout du compte niées des réalités parfaitement objectivables et importantes.

Le second est que l'adoption de modèles à au moins trois strates (e.g. signification, profil, sens) permet au contraire de rendre justice à la réalité sémantique des interprétationstypes ou emplois-types ${ }^{40}$ sans avoir ni à ignorer leur présence ni à les projeter dans la signification morphémique.

Le troisième est qu'en ce qui concerne les points de vue, le sémanticien peut se retrouver à devoir choisir en quelque sorte entre deux maux puisque s'il parait établi à ce stade des investigations empiriques que des points de vue objectivables sont associés aux emplois- 
types, autrement dit au niveau ultime de profilage de l'interprétation, il risque en les identifiant avec une signification codée qui serait partagée par tous les emplois d'un morphème, de ne pouvoir convaincre ni de la réalité de celle-ci ni de l'importance linguistique indiscutable par ailleurs des points de vue.

Or tout modèle plus fortement plurisémique échappe à cette impasse, dès lors qu'il est à la fois possible pour isoler la signification de ce morphème de considérer les points de vue comme défaisables ${ }^{41}$, et de considérer par ailleurs comme obligatoire la présence d'une étape de profilage argumentatif de l'interprétation. Ce qui revient à dire que tous les mots sont porteurs de points de vue, mais que la nature de ces points de vue n'est pas forcément déterminée par le morphème, en tous les cas pas par le morphème seul.

Or, la sémantique que Pierre-Yves Raccah a élaborée étant une sémantique des mots, il est important qu'elle soit une sémantique des points de vue et non une sémantique de la signification morphémique, puisque comme nous l'avons vu le niveau des mots est celui de la lexicalisation des points de vue ${ }^{42}$.

Reste néanmoins à signaler que le lien avéré entre points de vue et emplois-types par exemple n'implique pas en réalité que les points de vue soient circonscrits à ceux-ci et qu'il n'y ait aucun point de vue (ou contribution à la construction des points de vue) dans la signification morphémique. Ce qui importe en effet est que les méthodes utilisées pour isoler les uns et les autres puissent le cas échéant être différentes, le sémanticien étant en ce qui concerne le sens dans la position d'un guitariste pour qui un accord est à la fois et inséparablement un objet sonore identifiable en tant que tel et une forme spécifique d'utilisation d'un ensemble de cordes et de chacune de ces cordes, sachant que si l'objet sonore est accessible à l'auditeur, le geste lui ne l'est pas.

\section{Conclusion}

L'alternative entre descriptivisme et ascriptivisme n'est pas récente en sémantique mais comme nous l'avons vu, les rapports au monde faisant partie intégrale du monde, une telle alternative doit être dépassée. Car si de nombreux sémanticiens sont encore réticents à l'idée que les rapports au monde comme points de vue et les points de vue comme rapports au monde sont une part inévitable de toute description du monde, il est nécessaire de souligner ce que l'existence du syndrome du martin-pêcheur implique du point de vue théorique comme descriptif pour toute théorie sémantique.

Ce syndrome veut en effet que les poissons qu'un martin-pêcheur voit dans l'eau ne se trouvent pas là où il les voit, du fait de la diffraction de la lumière quand elle pénètre dans l'eau. Moyennant quoi, l'oiseau est donc équipé en quelque sorte pour corriger automatiquement ce qu'il perçoit et pour pouvoir situer la localisation véritable de ses proies.

157 Ce qu'implique une telle réalité est que face à l'alternative d'une langue sans points de vue (ni diffraction) rêvée par certains et d'une langue organisée autour de l'expression de points de vue, il n'y pas à choisir dès lors que :

- le monde est précisément ce qui se cache derrière les points de vue ;

- les langues n'ont pas la capacité de rendre les points de vue invisibles ;

- comprendre le(s) point(s) de vue adopté(s) est indispensable pour accéder à ce qu'il(s) voile(nt). 


\section{BIBLIOGRAPHIE}

Borg Emma, Minimal Semantics, Oxford, Clarendon Press, 2004.

Cadiot Pierre et Nemo François, « Propriétés extrinsèques en sémantique lexicale », Journal of French Language Studies $\mathrm{n}^{\circ}$ 7, 1997, pp. 1-19.

Conein Bernard, « Les sens sociaux : coordination de l'attention et interaction sociale, Intellectica vol. 1-2, $\mathrm{n}^{\text {os }} 26-27,1998$, pp. 181-202.

Furberg Mats, Saying and meaning, Oxford, Basil Blackwell, 1963.

Fodor Jerry et Lepore Ernest, « The Emptiness of the Lexicon: Reflections on James Pustejovsky's The Generative Lexicon », Linguistic Inquiry vol. 29, n² 2, 1998, pp. 269-288.

Grice Paul, « Logic and conversation », in Cole Peter et Morgan Jerry L. (eds), Syntax and semantics: Speech acts, New York, Academic Press, 1975.

Gosselin Laurent, Sémantique de la temporalité en français, Louvain-la-Neuve, Duculot, 1996.

Jacques Francis, L'Espace logique de l'interlocution, Paris, PUF, 1985.

Nemo François, « Contraintes énonciatives et argumentativité », Semantikos vol. 9, nº 2, 1985, pp. 21-34.

Nemo François, «Contraintes de pertinence et compétence énonciative : l'image du possible dans l'interlocution ", Thèse de l'EHESS, Paris, 1992.

Nemo François, «A description of argumentative relevance ", in Van Eemeren et alii (eds), Analysis and Evaluation. Proceedings of the Third ISSA Conference on Argumentation 2, Amstersdam, 1995, pp. 226-232.

Nemo François, « Making A difference Or Not : Utterances And Argumentation », in ISSA Proceedings, Amsterdam, 1998, pp. 594-598.

Corela, HS-19 | 2016 
Nemo François, « The Pragmatics of Signs, The Semantics of Relevance, and The Semantic / Pragmatic Interface », The Semantics-Pragmatics Interface from Different points of View, Amsterdam, Elsevier Science, 1999, pp. 343-417.

Nemo François, « Pour une approche indexicale (et non procédurale) des instructions sémantiques ", Revue de Sémantique et de Pragmatique ${ }^{\text {os }}$ 9-10, 2001, pp. 195-218.

Nemo François, But (and mais) as morpheme(s), DELTA vol. 18, São Paulo, 2002, pp. 87-114.

Nemo François, « Indexicalité, unification contextuelle et constitution extrinsèque du référent », Langages $\mathrm{n}^{\circ}$ 150, 2003, pp. 88-105.

Nemo François, «The Pragmatics of Common Ground. From Common knowledge to Shared Attention and Social Referencing ", in Fetzer Anita et Fischer Kerstin (eds), Lexical Markers of Common Grounds (Studies in Pragmatics, vol. 3), Amsterdam, Elsevier Science, 2007, pp. 143-158.

Nemo François, « Reconsidering the Discourse Marking Hypothesis ", in Celle Agnès et Huart Ruth (eds), Connectives As Discourse Landmark, Amsterdam, John Benjamins, 2007, pp. 195-210.

Nemo François, «Profilage temporel dans l'interprétation des morphèmes : de toujours à tout ", Revue de Sémantique et Pragmatique ${ }^{\text {os }}$ 25-26, 2009, pp. 97-120.

Nemo François, « Écart lexical ou résurgence morphémique? Approche linguistique des métaphores ", in Véronique Alexandre Journeau, Violaire Anger, Florence Lautel-Ribstein, Laurent Mattiussi (eds.), Métaphores et cultures. En mots et en images, Paris, L'Harmattan, 2012, pp. 31-50.

Nemo François, « Plurisémie et argumentation entre signification morphémique et signification lexicale », in Cozma Ana-Maria, Bellachhab Abdelhadi et Pescheux Marion (eds.), Du sens à la signification. De la signification au sens, Bruxelles, Peter Lang, 2014, pp. 301-312.

Nemo François et Létang Camille, « Post-Gricean Pragmatics and the Utterance / Contribution Distinction », Lodz Papers in Pragmatics, Berlin, Mouton de Gruyter, à paraître 2016.

Nemo François, Létang Camille et Petit Mélanie, « Prosodic constraints on argumentation : from individual utterances to argumentative exchanges », in Dima Mohammed et Lewinski Marcin (eds), Argumentation and Reasoned Action: Proceedings of the first European Conference on Argumentation. London, College publication, vol. 1, à paraître en mai 2016.

Nemo François et Petit Mélanie, « De la prosodie en discours à la prosodie en langue : lexicalisation de la forme prosodique des emplois-types ", in Yoo Hi-Yon et Delais-Roussarie Elisabeth (eds), Actes IDP 09 (Interface Discours \& Prosodie) Paris, 2009, pp. 302-312.

Nemo François et Petit Mélanie, « Sémantique des contextes-types », in De Saussure Louis et Rihs Alain (eds), Études de sémantique et pragmatique françaises, Berne, Lang, 2012, pp. 379-397.

Nemo François et Petit Mélanie, « Prosodie non-structurale et plurisémie », Revue de Sémantique et Pragmatique $\mathrm{n}^{\circ}$ 37, 2015, pp. 85-102.

Nemo François, Petit Mélanie et Portuguès Yann, « Profilage sémantique et plurisémie », Revue de Sémantique et Pragmatique ${ }^{\circ}$ 31, 2012, pp. 7-24.

Nowell-Smith Patrick, Ethics, Pelican, Harmondsworth (Middlesex), 1954.

Nowell-Smith Patrick, «Contextual implications and ethical theory », Proceedings of the Aristotelician Society Supll n ${ }^{\circ}$ 6, 1962, pp. 1-18.

Petit Mélanie, Nemo François et Létang Camille, « Prosodic constraints on pragmatic interpretation : a new chapter in linguistic pragmatics ", Lodz Papers in Pragmatics, Berlin, Mouton de Gruyter, à paraître en 2016. 
Pille Laetitia, « Une approche empirique de la sémantique du grec ancien permettant de révéler les idéologies sous-jacentes à l'utilisation de modèles mathématiques pour décrire les phénomènes musicaux : le cas des opposés barus et oxus ", Thèse de Doctorat, Université d'Orléans, 2015.

Rabatel Alain, La construction textuelle du point de vue, Lausanne, Delachaux et Niestlé, 1998.

Raccah Pierre-Yves, « Argumentation and natural language: presentation and discussion of four foundational hypotheses ", Journal of Pragmatics vol. $24 \mathrm{n}^{\text {os }}$ 1-2, 1995, pp. 1-15.

Raccah Pierre-Yves, «L'argumentation sans la preuve : prendre son biais dans la langue ", Cognition et Interaction vol. 2, $\mathrm{n}^{\text {os }} 1-2,1998, \mathrm{pp} .1-16$.

Raccah Pierre-Yves, « Lexique et idéologie : les points de vue qui s'expriment avant qu'on ait parlé ", in Carel Marion (éd.), Les facettes du dire : Hommage à Oswald Ducrot, Paris, Kimé, 2002, pp. 241-268.

Raccah Pierre-Yves, « Polyphonie et argumentation : des discours à la langue (et retour...), in Simonffy Zsuzsa (éd.), L'un et le multiple, Budapest, Tinta Könyvkiadó, 2006, pp. 120-152.

Raccah Pierre-Yves, « Explication et argumentation », in Hudelot Christian, Salazar Orvig Anne et Veneziano Edy (eds), L'explication. Enjeux cognitifs et interactionnels, Paris, Peeters, 2008, pp. 41-51.

Raccah Pierre-Yves, « Racines lexicales de l'argumentation : la cristallisation des points de vue dans les mots ", Verbum vol. 32, n 1, 2011, pp. 119-141.

Raccah Pierre-Yves, « Points de vue, idéologie et manipulation », in Raccah Pierre-Yves (dir). L'inscription des biais socio-cognitifs dans la langue, Limoges, Lambert-Lucas, 2014a, pp. 11-34.

Raccah Pierre-Yves, «Sémantique des points de vue et contraintes sur les possibles argumentatifs ", in Cozma Bellachhab et Pescheux Marion (dir.), Du sens à la signification De la signification aux sens, Bruxelles, New York, Peter Lang, 2014b, pp. 277-300.

Raccah Pierre-Yves, « Linguistic argumentation as a shortcut for the empirical study of argumentative strategies ", in Frans van Eemeren et Bart Garssen (eds), Reflections on Theoretical Issues in Argumentation Theory, Heidelberg, Springer, 2015, pp. 279-293.

Sapir Edward, « Grading: a study in semantics », Philosophy of Science ${ }^{\circ}$ 11, 1944, pp. 93-116.

\section{NOTES}

1. Dans les modèles que j'ai utilisé pour rendre compte de la polycatégorialité de nombreux signes (Nemo 2002) ou encore des listèmes ou des métaphores (Nemo 2012), les mots (lexèmes) ne sont qu'une partie du lexique, les unités porteuses de signification étant des unités prégrammaticales, renommées morphèmes.

2. Pour une présentation à mi-chemin de cet effort, voir Raccah (1995).

3. Ce faisant, je serai conduit à vérifier à la fois l'observation de Pierre-Yves Raccah (2014b) selon laquelle dans les hommages, on est souvent conduit à présenter sa propre pensée, la conviction qui est aussi la sienne (Raccah 2015) qu'il existe différentes méthodes d'étude d'une réalité comme celle de l'inscription des biais sociocognitifs dans la langue (Raccah 2014a), notamment parce que ceux-ci laissent différentes traces dans les langues, et enfin à défendre ma propre conviction que multiplier les points de vue sur les points de vue ne peut qu'étayer le projet d'une sémantique des points de vue.

4. Au-delà de l'indexicalité des instructions sémantiques qui interdit comme j'ai pu le souligner (Nemo 2001) tout rapprochement de la notion d'instruction et de la notion de procédure. 
5. L'emploi ici du terme de descripteur est délibéré, la question n'étant pas de savoir si la langue décrit le monde ou pas mais de savoir si quand elle le fait elle ne le fait pas à partir du rapport au monde des uns et des autres.

6. Nous avions pu montrer par exemple que les emplois de «nuit » en français parlent beaucoup plus de ce que font les hommes la nuit (e.g. « la nuit va être longue » ou « bonne nuit») que de quelque chose qui existerait indépendamment d'eux. En tout état de cause, même pris comme un phénomène naturel (étoile du soir ou du matin), la nuit ne peut exister que par rapport à un observateur (fut-il virtuel).

7. L'intenté étant défini comme ce dont on parle indépendamment de ce dont en dit. Comprendre la nature de l'intenté est une composante à part entière de l'interprétation qui fonctionne précisément en miroir de la compréhension des points de vue inscrits ou lisibles dans ce qui est dit.

8. L'opposition entre sémantique vériconditionnelle et sémantique cognitive s'est longtemps résumé à mettre entre les mots et le monde le filtre de la cognition, seule forme de pollution humaine admise dans le rêve philosophique d'une langue construite sur le seul rapport signe / monde.

9. Une unité lexicale peut donc en théorie : i) être monosémique et plurisémique, autrement dit n'avoir qu'un emploi lexicalisé mais auquel sont associées plusieurs strates sémantiques; ii) polysémique et unisémique, autrement dit avoir plusieurs emplois lexicalisés ayant chacun un sens monostratique; iii) polysémique et plurisémique, autrement dit avoir $n$ emplois lexicalisés ayant chacun un sens de chacun un sens pluristratique; iv) monosémique et unisémique, autrement dit n'ayant qu'un emploi lexicalisé et ce sens étant unistratique.

10. Les contraintes contributionnelles de type maxime de quantité (Létang et Nemo à paraître) produisent des lectures restrictives des énoncés : l'énoncé « Niche dans les Alpes ", s'il est aussi une contribution, sera ainsi souvent être interprété «Niche seulement dans les Alpes» (ou encore « ne niche pas dans les non-Alpes »).

11. Pour une critique plus générale de la différence entre langage formel et langue, voir Raccah (2008).

12. Chacune ayant inévitablement vocation à "défendre" la strate sémantique dont la description constitue son socle empirique.

13. En d'autres termes, s'il est tout à fait légitime le cas échéant de se focaliser sur telle ou telle strate interprétative (e.g. l'interprétation vériconditionnnelle), aucune théorie sémantique digne de ce nom ne peut ne pas rendre explicite sa conception du rapport entre toutes les strates connues.

14. À comparer avec Borg (2004 : 139) «In order to learn a language in the first place [...] it may well be that a grasp of speaker intentions is necessary. Yet all that the modular approach theory is committed to claiming is that once this system is in place, that is once a lexical item has been acquired, then the use of this item no longer need rest on the recognition of any kind of speaker intention ".

15. La notion de communication ostensivo-inférentielle de Sperber et Wilson relève directement de la notion d'attention contrôlée mais ne se donne pas comme argumentative (Nemo 1999).

16. Voir Nemo (2007).

17. Pour une présentation plus complète des contraintes scalaires sur l'argumentation, voir Nemo $(1985,1995,1998)$.

18. Notion à laquelle j'ai consacré toute ma thèse (Nemo 1992).

19. Ce qui revient à dire qu'il y a par exemple plusieurs façons de présenter la quantité comme significative.

20. Par exemple pour /enfin/ l'indication du fait qu'un problème se pose à un moment $t$ et est résolu à un moment $\mathrm{t}+1$. 
21. Par exemple, pour les emplois « constatifs» de /enfin/ (e.g. l'emploi de soulagement), le fait que $t$ et $t+1$ seront interprétés comme deux moments antérieurs à l'énonciation, le « enfin » étant associé à des situations où un problème qui se posait vient d'être résolu. Alors que pour l'emploi directif de «enfin» (i.e. taisez-vous enfin!), t sera le moment de l'énonciation et la résolution exigée du problème ne pourra se faire que dans un futur (voulu proche).

22. Par exemple dans les situations où le problème est résolu au moment de l'énonciation, le profilage prosodique concernera la nature du commentaire prosodique $\pi$ associé à «enfin », qui exprimera (Petit 2009) soit un soulagement (enfin « ouf») soit une irritation résiduelle (enfin «c'est pas trop tôt »). C'est ce niveau de profilage prosodique, nommé emploi-type, qui semble constituer le niveau ultime du lexique, autrement dit le niveau observable empiriquement et mémorisable par les sujets parlants.

23. Ainsi, une même contrainte - connue par la maxime de quantité de Grice (1975) mais formulée par Furberg (1963) à la suite de Nowell-Smith $(1954,1962)$ - produira à la fois les implicatures qui l'ont rendu célèbre et la lecture restrictive des énoncés ( $\mathrm{X}$ niche dans les Alpes » voulant dire « $\mathrm{X}$ ne niche que dans les Alpes») qui se retrouve dans l'interprétation de rouge-gorge (« la gorge (et pas la non-gorge) est rouge »).

24. Pierre-Yves Raccah (1998) a pu démontrer sur ce même terrain le fait qu'un mensonge n'était pas forcément faux (et inversement).

25. Le débat sur le rôle des langues dans le modelage de la perception humaine est en ce sens un faux débat, puisque la question ne concerne pas en réalité la perception des choses mais la forme d'attention qui leur est prêtée. Et qu'il est tout à fait clair que les langues modèlent bien les patterns attentionnels : le fait qu'un locuteur Palikur ait, avant de pouvoir dire « un » à propos de quelque chose, à se poser des tas de questions, et pour y répondre, à devoir prêter attention dans le monde à des choses très spécifiques auxquelles un locuteur du français ne prêtera aucune attention relève directement d'un modelage des patterns attentionnels.

26. On sait que la découverte $d u$ "langage des abeilles » a conduit les linguistes à faire pour les mêmes raisons de «la double articulation» le propre du langage humain, faute de pouvoir s'appuyer sur d'autres caractéristiques.

27. Avec $\mathrm{X}$ le langage, $\mathrm{Y}$ l'homme et $\mathrm{P}$ « être le propre de ».

28. On parle classiquement de présupposés existentiels et ils sont toujours écrits à gauche de la prédication.

29. De même, si on découvrait que le langage n'est pas le propre de l'homme moderne, l'énoncé «le langage est le propre de l'homme» pourrait rester vrai mais avec un changement d'interprétation de «l'homme». Pour le dire autrement, si la valeur de vérité de l'énoncé n'aurait pas changé, « ce qui le rend vrai » aurait lui changé.

30. Le syntagme "female bachelor» est parfois utilisé en anglais. La traduction française de « bachelor " par « célibataire » pose encore plus de problèmes puisque rien en français ne permet de réduire l'emploi aux seuls mâles, l'interprétation de l'exemple pour être analytique implique d'interpréter « hommes » comme « humains ».

31. On notera que dans la formulation (par ailleurs conventionnelle) que je viens d'employer, «ne pas vivre seul » est interprété comme « vivant une vie de couple » et ne s'applique donc pas à un homme qui vivrait avec ses parents par exemple, ce qui est inexplicable en termes de conditions de vérité.

32. On trouve parfois une compréhension partielle de la différence de points de vue « La nuit du 31 décembre 1999 au $1^{\text {er }}$ janvier 2000 il n'y aura pas grand-chose à célébrer; seul fait remarquable : les quatre chiffres de l'année commençante changeront tous. Dernière nuit d'une année, elle ne sera pas la dernière nuit du millénaire. Pas même la dernière d'un siècle. Si la première année de notre ère s'est terminée le 31 décembre de l'an 1 , il est clair que le premier siècle s'est terminé le 31 décembre de l'an 100 et non pas la dernière nuit de l'an 99. Le changement de siècle et de millénaire aura donc lieu dans la nuit de la Saint-Sylvestre unissant 
l'année 2000 au jour de l'an de l'année 2001. Que faire ? Célébrer en l'an 2000 un faux millénaire agrémenté d'un joli chiffre rond ou, en l'an 2001, un vrai millénaire à la rondeur hélas ébréchée?». (Wagensberg 1997, La Recherche, 90). À noter que si le changement des quatre chiffres a été noté, celui du seul chiffre des milliers ne l'a pas été spécifiquement.

33. Point de vue traduisant le fait que sa première pensée en matière de dangerosité des armes en question a été de penser au danger qu'elle représente pour les policiers et non pour les nonpoliciers. Ce qui est contraire à la définition du rôle de la police mais aussi cohérent avec le fait que l'énonciateur soit un syndicaliste.

34. AP veut dire « Avant le Présent ».

35. À noter que le choix de «into" met quant à lui en avant la question du peuplement de «l'intérieur des Amériques » et ce dans un contexte où les traces certaines les plus anciennes de peuplement des Amériques se trouvent en réalité près des côtes au sud de l'Amérique du sud (Chili) et plaident donc pour une descente assez rapide le long des côtes.

36. Ce que l'on peut traduire en français par «Un modèle basé sur l'ADN mitochondrial des amérindiens propose une migration vers la Béringie entre $30 \mathrm{k}$ et $25 \mathrm{k}$ années $\mathrm{BP}$, et une migration vers les Amériques qui se produit autour de $10 \mathrm{k}$ à $15 \mathrm{k}$ ans après l'isolement de la petite population fondatrice ». À noter que le terme « Native Americans » a lui aussi subi une restriction devenue tellement conventionnelle qu'il ne peut aujourd'hui s'appliquer qu'aux américains « dont les ancêtres sont nés en Amérique avant l'arrivée des Européens en 1492 ». Ceci très loin du simple croisement des deux propriétés « être américain » et « être natif de quelque part » en un prédicat « être natif de l'Amérique ».

37. Ce moyen peut être linguistique (e.g. prosodique ou lexical) mais il peut aussi ne pas l'être. Parce que pendant longtemps il ne l'a pas été, on peut dire que le langage poursuit avec des moyens radicalement nouveaux un jeu (manipulatoire) qui l'a précédé de beaucoup.

38. Le problème n'est pas un problème de polysémie : un homme de 80 ans pouvant parfaitement dire «je vous présente mes enfants» en parlant de personnes de 55 et 58 ans. En ce sens, " enfant» ne s'oppose donc pas en général et en langue à "adulte». Ce qui est pertinent en revanche, ce sont les propriétés extrinsèques pertinentes dans le contexte, à savoir ici le fait que les enfants sont censés suivre leurs parents (ou des adultes) et que les enfants en ce sens-là ne peuvent pas être des adultes. Ce qui est précisément ce que présuppose doublement notre énoncé quand il tient pour acquis que les enfants dont il s'agit vont suivre les adultes qu'ils accompagnent.

39. Au sens proposé par Pierre-Yves Raccah, «partagée » n’impliquant pas «faisant consensus » mais « connu par delà les divergences éventuelles ».

40. Qui représentent en l'occurrence deux niveaux de profilage distincts.

41. Démarche que j'ai adoptée à partir de 1999 pour la description de tous les morphèmes sur lesquels j'ai travaillé, à savoir [but] et [even] en Anglais (Nemo 2002, 2006a, 2007) ; /table/ (Nemo 2003) ; [enfin], [encore] (Nemo 2001) ; [tou(s/t)] (Nemo 2009).

42. Voir Raccah (2011).

\section{RÉSUMÉS}

Cet article entend montrer en écho aux travaux de Pierre-Yves Raccah, l'existence générale d'une strate interprétative spécifique, linguistiquement construite pour une large part, et qui concerne le marquage et la lexicalisation des points de vue et rapports au monde. Après avoir rappelé le 
rapport entre polysémie et plurisémie comme diversité des strates interprétatives associées à chaque emploi d'un signe, introduit les strates argumentatives liées à l'attention contrôlée et à la contrainte de scalarité ainsi que les contraintes que la prosodie impose aux points de vue exprimés, il vise à démontrer que ceux-ci sont présents en réalité dans la détermination de la référence des syntagmes nominaux. Est notamment démontré par un ensemble d'exemples très variés que l'interprétation extensionnelle d'un tel élément, autrement dit la définition de ce à quoi il s'applique, s'avère être fonction de la prédication dans laquelle il est enchâssé, au point que l'énoncé négatif s'avère ne pas nier la même chose que ce qui est présent dans l'énoncé positif correspondant. Dans tous les cas étudiés, ces phénomènes de restriction sémantique s'avèrent ancrés dans des façons particulières de regarder le monde. Ce qui impose de dépasser l'alternative d'une langue sans points de vue rêvée par certains et d'une langue organisée autour de l'expression de points de vue, dès lors que le monde n'étant accessible que par le truchement de points de vue, et les langues n'ayant pas la capacité de rendre les points de vue invisibles et comprendre ceux-ci étant indispensable pour accéder à ce qu'ils voilent.

The aim of this paper is to show, in relation with Pierre-Yves Raccah's work, the general existence of a specific interpretative layer, linguistically built to a large extent and which concerns the marking and lexicalization of viewpoints. After recalling the distinction between polysemy and plurisemy (defined as the multi-layered interpretation associated with any use of a word), the argumentative layers at stake (associated with the mechanism of controlled attention and the constraint of scalarity), and the impact of prosody on viewpoints are presented. It is then shown that viewpoints are not limited to argumentative layers and play a crucial role in the determination of the reference of noun phrases. Importantly, is demonstrated on a wide range of examples that the extensional interpretation of such elements is indeed produced by the predicative element (to the point that a negated predicate can be proven to negate something different that what is found in its positive counterpart). Furthermore, in all the examples which are provided, such "semantic restrictions" by the predicate appear to be anchored into specific ways to see the world. This imposes to overcome the alternative between a perspective-free language / world relationship and a world-free language / viewpoint relationship. The world is what is behind the way we access to it, and because languages do not have the capacity to make such viewpoints invisible, they are fully part of the interpretation process, for understanding them is compulsory to have access to what they veil.

\section{INDEX}

Mots-clés : points de vue, plurisémie, restriction sémantique, référence des syntagmes nominaux, strates interprétatives, syndrome du martin-pêcheur.

Keywords : viewpoints, plurisemy, semantic restriction, reference of noun phrases, interpretative layers, kingfisher's syndrome.

\section{AUTEUR}

\section{FRANÇOIS NEMO}

Université d'Orléans, LLL, UMR7270 